\title{
An uncommon clinical picture: Wellens' syndrome in a morbidly obese young man
}

\author{
Salvatore Corrao $\cdot$ Salvatore Amico $\cdot$ \\ Luigi Calvo · Eliana Barone · Giuseppe Licata
}

Received: 9 December 2009/ Accepted: 3 February 2010/Published online: 30 March 2010

(C) SIMI 2010

A 39-year-old man presented to the emergency department (ED) of the "Paolo Giaccone" Academic Hospital, Palermo (Italy). He had anterior chest pain that did not radiate to the neck or arms. The patient came from home where the chest pain initiated. The patient was morbidly obese (BMI $54 \mathrm{~kg} / \mathrm{m}^{2}$ ). At the ED, the patient's blood pressure was $120 / 80 \mathrm{mmHg}$, the serum troponin I concentration was $0.029 \mathrm{ng} / \mathrm{ml}$ (normal values $<0.034$, borderline 0.034-0.12), myoglobin $45 \mathrm{ng} / \mathrm{ml}$ (normal values $<120$ ). While experiencing chest pain, the patient underwent a standard 12 lead electrocardiogram (ECG) that was normal. An echocardiogram, also during the chest pain, excluded the presence of hypo-akinetic left ventricle areas. He was admitted to the internal medicine ward of the same hospital for clinical monitoring. Cardiac biohumoral markers were measured every $4 \mathrm{~h}$, and were always within normal range (only troponin I reached $0.12 \mathrm{ng} / \mathrm{ml}$ falling to $0.028 \mathrm{ng} / \mathrm{ml}$ ). On the following morning, anterior chest pain recurred. Another ECG was performed, but showed no abnormalities (Fig. 1a). During the afternoon, the patient underwent another ECG. He had no thoracic discomfort or pain, but the ECG showed a biphasic T wave inversion in V2-V6 precordial leads (Fig. 1b). Cardiac markers resulted again within normal range. An internal medicine resident who was trained to manage uncommon clinical pictures by PubMed searching [1] recognized this abnormal pattern

S. Corrao $(\bowtie) \cdot$ S. Amico · L. Calvo · E. Barone · G. Licata Dipartimento Biomedico di Medicina Interna e Specialistica, Università di Palermo, Piazza delle Cliniche, 2,

90127 Palermo, Italy

e-mail: s.corrao@tiscali.it
(ECG abnormalities in an asymptomatic patient with prior anterior chest pain) performed a PubMed search using the following simple search string (biphasic $\mathrm{T}$ waves AND precordial leads). Six citations were found and Wellens' Syndrome diagnosis was suggested. An internist confirmed the diagnostic hypothesis, and the patient, was taken to the invasive hemodynamic lab. Coronary arteriography was performed showing a 95\% stenosis of the left anterior descending (LAD) coronary artery (Fig. 2a). The patient underwent percutaneous transluminal coronary angioplasty with resolution of the stenosis (Fig. 2b) and was discharged after 6 days in good condition.

In 1982, Wellens et al. [2] described a characteristic ECG pattern associated with a critical stenosis of the LAD coronary artery and impending myocardial infarction. Tilkian [3] was the first to use the term Wellens' syndrome defined as a group of ECG signs that occur during the painfree period in a patient with unstable angina. These ECG abnormalities, in the absence of pathologic $\mathrm{Q}$ waves, are predictive of a critical proximal LAD stenosis [2, 4]. They consist of an isolectric or minimally elevated ST segment followed by a concave or straight ST segment and symmetrically inverted (or biphasic) $\mathrm{T}$ waves in the precordial leads, frequently in V2-V3, but sometimes involving V4, V5 or V6. [4]. This was the case of our patients who showed a biphasic $\mathrm{T}$ wave inversion in V2-V6, leads during a pain-free period.

In conclusion, this uncommon clinical picture involves one of the four clinical diagnostic strategies known as pattern recognition. This kind of diagnostic method particularly depends upon the experience of the physician. Nevertheless, we think this kind of clinical picture could be interpreted successfully using PubMed by a non-experienced physician [5]. 
Fig. 1 a A normal ECG during precordial chest pain.

b A biphasic $\mathrm{T}$ wave inversion in V2-V6 precordial leads in a pain-free period. This ECG also shows an acute $\mathrm{T}$ wave inversion in lead aVL that does not seem relevant in comparisons with the abovementioned precordial lead findings

Fig. 2 a The stenosis of the left anterior descending (LAD) coronary artery; the broken line arrow shows the beginning of vascular roughness, while the second arrow shows exact narrowing of the LAD coronary artery. b Resolution of the critical stenosis after PTCA
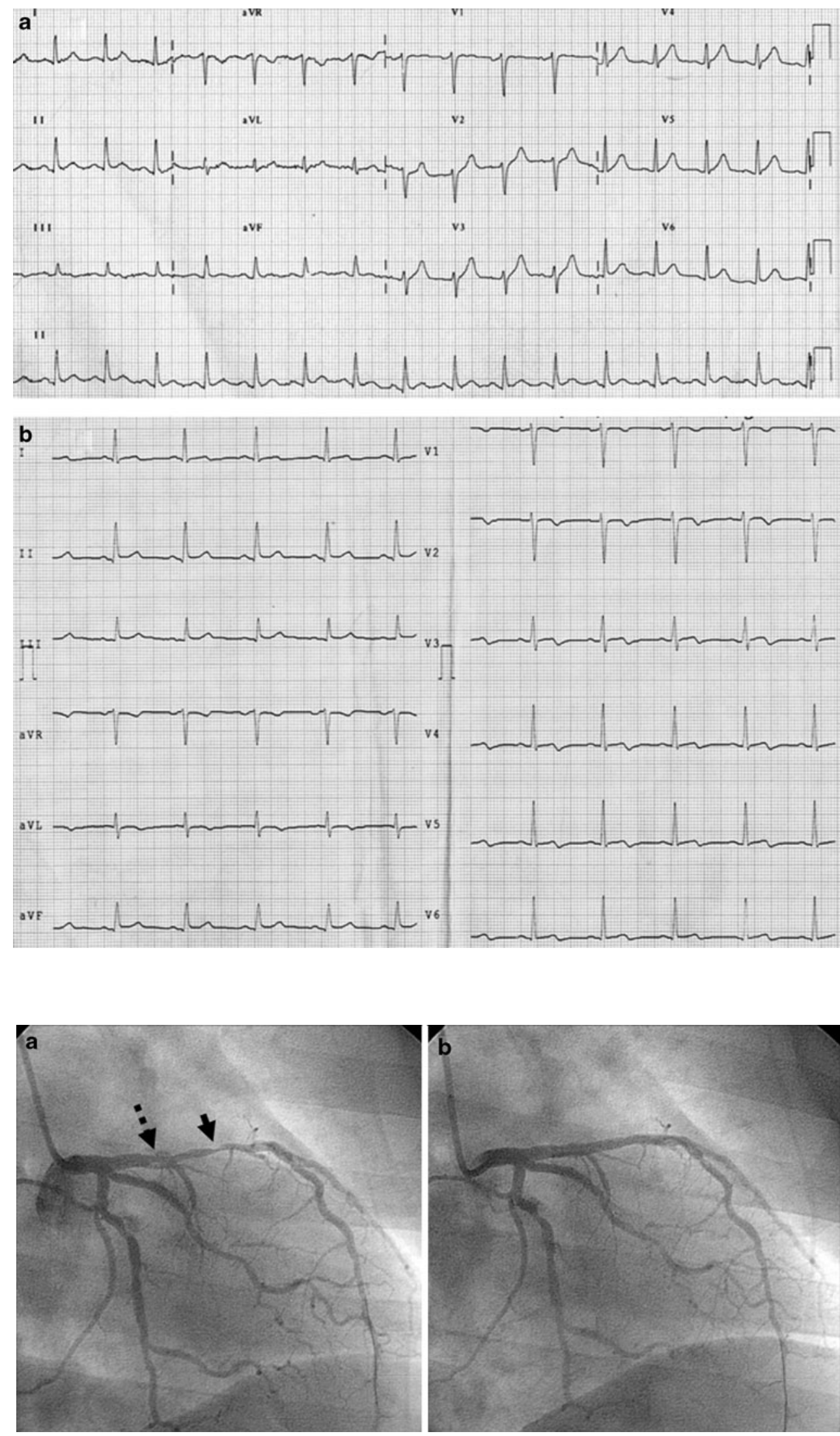


\section{Conflict of interest None.}

\section{References}

1. Corrao S, D'Alia R, Caputo S, Arnone S, Pardo GB, Jefferson T (2005) A systematic approach to medical decision-making of uncommon clinical pictures: a case of ulcerative skin lesions by palm tree thorn injury and a one-year follow-up. Med Inform Internet Med 30(3):203-210

2. De Zwaan C, Bar WHM, Wellens HJJ (1982) characteristic electrocardiographic pattern indicating a critical stenosis high in the left anterior descending coronary artery in patients admitted because of impending myocardial infarction. Am Heart J 103:730 736

3. Conover MB (1996) Understanding electrocardiography. Mosby, St. Louis, pp 344-361

4. De Zwaan C, Bar FW, Janssen JHA et al (1989) Angiographic and clinical characteristics of patients with unstable angina showing an ECG pattern indicating critical narrowing of the proximal LAD coronary artery. Am Heart J 117:657-664

5. Federspil G, Vettor R (2008) Rational error in internal medicine. Intern Emerg Med 3(1):25-31 